\title{
Oscillation Criteria for First Order Dynamic Equations with Maxima on Time Scales
}

\author{
H. A. Agwa ${ }^{1}$, Esraa Magdy ${ }^{2}$ \\ ${ }^{1}$ Faculty of Education/Department of Mathematics/Pure Mathematics, Ain Shams University, Cairo, Egypt \\ ${ }^{2}$ Faculty of Education/Department of Mathematics/Pure Mathematics, Ain Shams University, Cairo, Egypt \\ *Corresponding Author: H. A. Agwa, Faculty of Education/Department of Mathematics/Pure \\ Mathematics, Ain Shams University, Cairo, Egypt
}

Abstract: In this work, we establish some new oscillation results for the first order dynamic equations with maxima

$$
\begin{gathered}
x^{\Delta}(t)+q(t) \max _{s \in[t-\delta, t]} x(s)=0, \\
x^{\Delta}(t)+p_{1}(t) \max _{s \in\left[\tau_{1}(t), t\right]} x(s)-p_{2}(t) \max _{\left.s \in\left[\tau_{2}(t), t\right)\right]} x(s)=0,
\end{gathered}
$$

and

$$
x^{\Delta}(t)+\sum_{i=1}^{n} p_{i}(t) \max _{s \in\left[\delta_{i}(t), \sigma(t)\right]} x(s)+\sum_{j=1}^{m} q_{j}(t) x\left(\eta_{j}(t)\right)=0,
$$

Our results not only complement and generalize some existing results, but also can be applied to some oscillation problems that were not covered before, we also give some examples to illustrate our main results

Keywords: Oscillation, dynamic equations, time scales, maxima.

\section{INTRODUCTION}

The theory of time scales was introduced by Hilger [1] in order to unify, extend and generalize

ideas from discrete calculus, quantum calculus, and continuous calculus to arbitrary time scale calculus. A time scale $\mathrm{T}$ is a nonempty closed subset of the real numbers $\mathrm{R}$. When the time scale equals to the real numbers or integer numbers, the obtained results represent the classical theories of the differential and difference equations. Many other interesting time scales exist and give arise to many applications. The new theory of the so - called " dynamic equation" not only unify the theories of differential equations and difference equations, but also extends these classical cases to the so called $\mathrm{q}$ - difference equations (when $\mathrm{T}=\mathrm{q}^{\mathrm{No}}:=\left\{\mathrm{q}^{\mathrm{t}}: \mathrm{t} \in\right.$ No for $\left.\mathrm{q}>1\right\}$ or $\mathrm{T}=\mathrm{q}^{\mathrm{Z}}=\mathrm{q}^{\mathrm{Z}} \mathrm{U}\{0\}$ ) which have important applications in quantum theory see [2]. Also, it can be applied on different types of time scales like $\mathrm{T}=\mathrm{hZ}$ and $\mathrm{T}=\mathrm{N}_{0}{ }^{2}$. For an introduction to time scale calculus and dynamic equations, see Bohner and Peterson books [3, 4].

We note that equations with maxima occur in the problem of automatic regulation of various real system is considered in [5]. The maxima arise when the regulation law corresponds to the maximal deviation of the regulable quantity. The paper in which oscillatory properties of equations with maxima are considered in [6].

In this paper, we present time scale analogies of some result for continuous case $T=R$ and the discrete case $T=Z$. A nontrivial solution of the following equations is said to be oscillatory if it is neither eventually positive nor eventually negative. Otherwise, the solution is called nonoscillatorv. A solution is called z-type if it is either nonpositive or nonnegative.

Next, let us recall some known oscillation results on this subject. The first order delay differential equation

$$
x^{\prime}(t)+q(t) \max _{s \in[t-\delta, t]} x(s)=0,
$$


equation (1) is nonoscillatory if $q(\mathrm{t})$ is of one sign as in [7 ] and if $\mathrm{q}(\mathrm{t})$ is oscillatory, then equation (1) has at least one nonoscillatorv solution. For multidelays H. A. Agwo considered in [8] the following equation

$y^{\Delta}(t)+\sum_{i=1}^{n} p_{i}(t) y\left(\tau_{i}(t)\right)=0$,

Where

(i) $\mathrm{p}_{\mathrm{i}}(\mathrm{t}), \mathrm{i}=1,2, \ldots \mathrm{n}$, are nonnegative real valued rd-continuous function defined on $\mathrm{T}$,

(ii) $\mathrm{T}_{\mathrm{i}}():. \mathrm{T} \rightarrow \mathrm{T}$ and $\tau_{i}(t)<t$, for all $\mathrm{t} \in T, \mathrm{i}=1,2, \ldots \mathrm{n}$.

Then equation (2) is oscillatory if

$$
\lim _{t_{0 \rightarrow \infty}} \sup _{t>t_{0}} \sup _{\lambda \in E}\left\{\frac{\lambda \sum_{i=1}^{n} p_{i}(t)}{\sum_{i=1}^{n} p_{i}(t) \exp \left[\left(\int^{t}-\int_{\tau_{i}(t)}^{t} \xi_{\mu(s)}\left(-\lambda \sum_{i=1}^{n} p_{i}(s)\right) \Delta s\right)\right.}\right\}<1,
$$

Where $\mathrm{E}=\left\{\boldsymbol{\lambda}>0: 1-\lambda \sum_{i=1}^{n} p_{i}(t) \mu(t)>0\right\}$. This result will be used in the proof of our main result.

\section{MAIn RESUltS}

\subsection{Theorem}

If $\mathrm{q}(\mathrm{t})$ is of one sign of equation

$\mathrm{x}^{\Delta}(\mathrm{t})+\mathrm{q}(\mathrm{t}) \max _{\mathrm{s} \in[\mathrm{t}-\delta, \mathrm{t}]} \mathrm{x}(\mathrm{s})=0,(3)$

then, every solution of (3) is nonoscillatory.

\section{Proof:-}

When $\mathrm{q}(\mathrm{t})=0$, then $x^{\Delta}(t)=0$ and consequently, $x(t)=x\left(t_{0}\right)=\alpha, \propto \in R$. Also when $\delta=0$, then equation (3) becomes $x^{\Delta}(\mathrm{t})+\mathrm{q}(\mathrm{t}) x(\mathrm{~s})=0$. This equation is not oscillatory. If $q(t)>0$, suppose that $x(\mathrm{t})=0$ is an oscillatory solution of $(3)$, then $x(\mathrm{t})$ is not $\mathrm{z}$-type solution and otherwise, $\mathrm{x}(\mathrm{t})=0$ eventually. Therefore, there exist $t_{1} t_{2} t_{3}$ such that $x\left(t_{1}\right)=x\left(t_{2}\right)=x\left(t_{3}\right)=0$ and $x(t)<0$.

For $t \in\left(t_{1}, t_{2}\right)_{T} x(t)>0$ for $t \in\left(t_{2}, t_{3}\right)_{T}$. Thus $x^{\Delta}\left(t_{2}\right)=-q\left(t_{2}\right) \max _{\left[t_{2}-\delta, t_{2}\right]} x(s) \leq 0$, which is a contradiction.

For $q(t)<0$, the proof is simillar, hence it's omitted.This completes the proof.

\subsection{Theorem}

Assume that we have

$\mathrm{x}^{\Delta}(\mathrm{t})+p_{1}(t) \max _{s \in\left[\tau_{1}(t), t\right]} x(s)-p_{2}(t) \max _{\left.s \in\left[\tau_{2}(t), t\right)\right]} x(s)=0$

(i) $\tau_{2}(t)>\tau_{1}(t), p_{1}, p_{2}>0$,

(ii) there exists $t_{1} \geq t_{0}$ such that

$$
p_{1}(t) \geq p_{2}\left(\tau_{2}^{-1}\left(\tau_{1}(t)\right)\right) \quad \text { for all } t \geq t_{1} \geq t_{0}
$$

(iii) $\int_{t_{0}}^{\infty} p_{2}(s) \Delta s<\infty, \liminf f_{t \rightarrow \infty} \int_{\tau_{1}(t)}^{t} p_{1}(s) \Delta s>c, c>0$.

Then every solution of equation (4) is either oscillatory or tends to zero.

Proof:

Assume that equation (4) has a nonoscillatory solution $x(\mathrm{t})$ on $\left[t_{0}, \infty\right)_{T}$. Suppose that there exists $t \geq \tau_{1}^{-1}\left(t_{1}\right)$.

Now, we consider the following two cases:

I. $x(\mathrm{t})$ is bounded on $\left[t_{1}, \infty\right)_{T}$

Let

$z=x-\int_{\tau_{2}^{-1}\left(\tau_{1}(t)\right)}^{t} p_{2}(s) \max _{\xi \in\left[\tau_{2}(s), s\right]} x(\xi) \Delta s .(5)$ 
Then,

$z^{\Delta}=x^{\Delta}-p_{2}(t) \max _{\xi \in\left[\tau_{2}(t), t\right]} x(\xi)+p_{2}\left(\tau_{2}^{-1}\left(\tau_{1}(t)\right)\right) \max _{\xi \in\left[\tau_{1}(t), \tau_{2}^{-1}\left(\tau_{1}(t)\right)\right]^{x}} x(\xi)$.

From (4) and (6) we have

$z^{\Delta}(t)+p_{1}(t) \max _{\xi \in\left[\tau_{1}(t), t\right]} x(\xi)-p_{2}\left(\tau_{2}^{-1}\left(\tau_{1}(t)\right)\right) \max _{\xi \in\left[\tau_{1}(t), \tau_{2}^{-1}\left(\tau_{1}(t)\right)\right]} x(\xi)=0$,

since $\tau_{1}(t)<\tau_{2}(t), x(t)>0$, then we have

$$
\max _{\xi \in\left[\tau_{1}(t), t\right]} x(\xi)>\max _{\xi \in\left[\tau_{1}(t), \tau_{2}^{-1}\left(\tau_{1}(t)\right)\right]} x(\xi) .
$$

Due to (7) and condition (ii), we get $z^{\Delta}(t) \leq 0$ for all $t>\tau_{1}^{-1}\left(t_{1}\right)$ and consequently $z(\mathrm{t})$ is nonincreasing on $\left[t_{1}, \infty\right)_{T}$. Since $\int_{t_{0}}^{\infty} p_{2}(s) \Delta s<\infty$, and $\mathrm{x}(\mathrm{t})$ bounded then

$$
\int_{\tau_{1}^{-1}(t)}^{\infty} p_{2}(t) \max _{\xi \in\left[\tau_{2}(s), s\right]} x(\xi) \Delta s<\infty,
$$

then,

$\lim _{t \rightarrow \infty} \int_{\tau_{2}^{-1}\left(\tau_{1}(t)\right)}^{\infty} p_{2}(t) \max _{\xi \in\left[\tau_{2}(t), t\right]} x(\xi) \Delta s=0$.

Hence $\mathrm{z}(\mathrm{t})$ is bounded on $\left[\tau_{1}^{-1}(t), \infty\right)_{T}$, so $\lim _{t \rightarrow \infty} z(t)$ exists.

Denote

$l=\lim _{t \rightarrow \infty} Z(t)$,

from (5), (8) and (9) we have

$\lim _{t \rightarrow \infty} z(t)=\lim _{t \rightarrow \infty} x(t)=l$,

where $l>0$ or $l=0$

If $l>0$, integrating (4) from $\tau_{1}(t)$ to $t$, we get

$$
\begin{array}{r}
x(t)-x\left(\tau_{1}(t)\right)+\int_{\tau_{1}(t)}^{t} p_{1}(s) \max _{\xi \in\left[\tau_{1}(s), s\right]} x(\xi) \Delta s-\int_{\tau_{1}(t)}^{t} p_{2}(s) \max _{\xi \in\left[\tau_{2}(s), s\right]} x(\xi) \Delta s=0, \\
t \geq \tau_{1}^{-2}\left(t_{1}\right) .
\end{array}
$$

From (10), there is a $t_{2} \geq \tau_{1}^{-2}\left(t_{1}\right)$ such that

$\frac{l}{2}<x(t)<2 l \quad$ for all $t \geq t_{2}$.

Hence, from (11), (12), we have

$x(t)-x\left(\tau_{1}(t)\right)+\frac{l}{2} \int_{\tau_{1}(t)}^{t} p_{1}(s) \Delta s-2 l \int_{\tau_{1}(t)}^{t} p_{2}(s) \Delta s<0$ for all $t \geq t_{2}$.

Taking the lower limit in (13) as $t \rightarrow \infty$,we get

$$
\int_{t-\tau_{1(t)}^{t}}^{t} p_{1}(s) \Delta s<0
$$

Which is a contradiction.

II. If $x(t)$ is unbounded on $\left[t_{1}, \infty\right)$ from (4), we have

$x^{\Delta}(t) \leq p_{2}(t) \max _{s \in\left[\tau_{2}(t), t\right]} x(s), t \geq \tau_{1}\left(t_{1}\right)$.

Integrating (14) from $\tau_{1}(t)$ to $t$, we get

$$
x(t) \leq x\left(\tau_{1}(t)\right)+\int_{\tau_{1}(t)}^{t} p_{2}(s) \max _{\xi \in\left[\tau_{2}(s), s\right]} x(\xi) \Delta s .
$$

Since $x(t)$ is positive and unbounded, we must have,

$$
x^{\Delta}(t)>0 \text { for } t \geq \tau_{1}^{-1}\left(t_{1}\right)
$$


and so,

$$
x(t) \leq x\left(\tau_{1}(t)\right)+\int_{\tau_{1}(t)}^{t} p_{2}(s) \Delta s
$$

By the Gronwall inequality

$$
x(t) \leq x\left(\tau_{1}(t)\right) e_{p_{2}}\left(t, \tau_{1}(t)\right)<\infty,
$$

which is a contradiction.

Assume that equation (4) has eventually negative solution $x(\mathrm{t})$ on $\left[t_{0}, \infty\right)_{T}$ then, there exists $t_{3} \geq$ $t_{0}$ such that $x(t)<0$ on $\left[\tau_{1}^{-1}\left(t_{4}\right), \infty\right)_{T}$.

Let $y=-x$, then equation (2.2) takes the form

$y^{\Delta}(t)+p_{1}(t) \min _{s \in\left[\tau_{1}(t), t\right]} y(s)-p_{2}(t) \min _{s \in\left[\tau_{2}(t), t\right]} y(s)=0$.

Assume that

$w(t)=y(t)+\int_{t}^{\infty} p_{2}(s) \min _{\xi \in\left[\tau_{2}(s), s\right]} y(\xi) \Delta s$,

then

$w^{\Delta}(t)=y^{\Delta}(t)-p_{2}(t) \min _{s \in\left[\tau_{1}(t), t\right]} y(s)$,

from (15),(17) we get

$w^{\Delta}(t)+p_{1}(t) \min _{s \in\left[\tau_{1}(t), t\right]} y(s)=0$.

Since $p_{1}(t), y(t)>0$, then $w^{\Delta}(t) \leq 0$.

Now, we consider the following two cases:

I. $\quad \mathrm{y}$ is bounded on $\left[\tau_{1}^{-1}\left(t_{3}\right), \infty\right)_{T}$.

Since $\int p_{2}(s) \Delta s<\infty$, y is bounded, then $\mathrm{w}$ is bounded, then $w$ is bounded $\left[\tau_{1}^{-1}\left(t_{3}\right), \infty\right)_{T}$.

Since $w$ is bounded and $w^{\Delta} \leq 0$, then. Hence.

$\lim _{t \rightarrow \infty} w(t)=l$.

From (16), we have $\lim _{t \rightarrow \infty} w(t)$ exists. $=\lim _{t \rightarrow \infty} y(t), y(t)>0$. Hence $l \geq 0$.

If $l>0$, then there exists $t_{4} \geq \tau_{1}^{-1}\left(t_{3}\right)$ such that

$\frac{l}{2}<y(t)<2 l$ for all $t \geq t_{4}$.

Integrating (18) from $\tau_{1}(t)$ to $t$ we get

$w(t)-w\left(\tau_{1}(t)\right)+\frac{l}{2} \int_{\tau_{1}(t)}^{t} p_{1}(s) \Delta s<0$.

Taking the lower limit as $t \rightarrow \infty$, we get

$$
\int_{\tau_{1}(t)}^{t} p_{1}(s) \Delta s<0
$$

which is a contradiction.

II. $\quad \mathrm{y}(\mathrm{t})$ is unbounded on $\left[\tau_{1}^{-1}\left(t_{3}\right), \infty\right)_{T}$.

From (15) we have

$y^{\Delta}(t) \leq p_{2}(t) \min _{s \in\left[\tau_{2}(t), t\right]} y(s)$.

Integrating (22) from $\tau_{1}^{-1}\left(t_{4}\right)$ to $t$, we get,

$$
y(t)-y\left(\tau_{1}^{-1}\left(t_{4}\right)\right) \leq \int_{\tau_{1}^{-1}\left(t_{4}\right)}^{t} p_{2}(s) \min _{\xi \in\left[\tau_{2}(s), s\right]} y(\xi) \Delta s .
$$


Let $\theta=\tau_{2}(s)$, then the above inequality can be written in the form

$y(t)-y\left(\tau_{1}^{-1}\left(t_{4}\right)\right) \leq \int_{\tau_{2}\left(\tau_{1}^{-1}\left(t_{4}\right)\right)}^{\tau_{2}(t)} p_{2}\left(\tau_{2}^{-1}(\theta)\right) \min _{\xi \in\left[\theta, \tau_{2}^{-1}(\theta)\right]} y(\xi) \Delta \theta$.

Since $>0$,y is unbounded, then $y^{\Delta}>0$, and consequently y is non-decreasing. So from (23), we have

$$
\begin{aligned}
y(t) & \leq y\left(\tau_{1}^{-1}\left(t_{4}\right)\right)+\int_{\tau_{2}\left(\tau_{1}^{-1}\left(t_{4}\right)\right)}^{\tau_{2}(t)} p_{2}\left(\tau_{2}^{-1}(\theta)\right) y(\theta) \Delta \theta \\
& \leq y\left(\tau_{1}^{-1}\left(t_{4}\right)\right)+\int_{\tau_{2}\left(\tau_{1}^{-1}\left(t_{4}\right)\right)}^{t} p_{2}\left(\tau_{2}^{-1}(\theta)\right) y(\theta) \Delta \theta,
\end{aligned}
$$

which implies that

$$
y(t) \leq y\left(\tau_{1}^{-1}\left(t_{4}\right)\right) e_{p_{2}}\left(t, \tau_{2}\left(\tau_{1}^{-1}\left(t_{4}\right)\right)\right)<\infty,
$$

which is a contradiction. the proof is complete.

\subsubsection{Remark}

If we choose $\tau_{1}(t)=t-\delta_{1}$ and $\tau_{2}(t)=t-\delta_{2}$ in the above theorem, then equation (4) takes the form

$x^{\Delta}(t)+p_{1}(t) \max _{s \in\left[t-\delta_{1}, t\right]} x(s)-p_{2}(t) \max _{s \in\left[t-\delta_{2}, t\right]} x(s)=0$

where

(i) $\delta_{1}>\delta_{2}, p_{1}, p_{2}>0$,

(ii) there exists $t_{1} \geq t_{0}$ such that

$p_{1}(t) \geq p_{2}\left(t-\left(\delta_{1}-\delta_{2}\right)\right)$ for all $t \geq t_{1} \geq t_{0}$.

(iii) $\int_{t_{0}}^{\infty} p_{2}(s) \Delta s<\infty$ and $\liminf _{t \rightarrow \infty} \int_{t-\delta_{1}}^{t} p_{1}(s) \Delta s>c, c>0$.

Then every solution of equation (24) is either oscillatory or tends to zero.

\subsection{Theorem}

Assume that

$\mathrm{x}^{\Delta}(\mathrm{t})+\sum_{i=1}^{n} p_{i}(t) \max _{s \in\left[\delta_{i}(t), \sigma(t)\right]} x(s)+\sum_{j=1}^{m} q_{j}(t) x\left(\eta_{j}(t)\right)=0$,

where

$\delta_{i}, \eta_{j}<t, i=1, \ldots n, j=1, \ldots m$, such that

I. $\lim _{t_{0 \rightarrow \infty}} \sup _{t>t_{0}} \sup _{\lambda \in E_{1}}\left\{\frac{\lambda \sum_{k=1}^{n+m} h_{k}(t)}{\sum_{k=1}^{n+m} h_{k}(t) \exp \left(\int^{2}-\int_{\tau_{k}}^{t}(t) \xi_{\mu(s)}\left(-\lambda \sum_{k=1}^{n+m} h_{k}(s)\right) \Delta s\right)}\right\}<1$,

II. $\lim _{t_{0 \rightarrow \infty}} \sup _{t>t_{0}} \sup _{\lambda \in E_{2}}\left\{\frac{\lambda \sum_{j=1}^{m} \frac{q_{j}(t)}{e_{p}\left(\eta_{j}(t), t\right)}}{\sum_{j=1}^{m} \frac{q_{j}(t)}{e_{p}\left(\eta_{j}(t), t\right)} \exp \left(t-\int_{\eta_{j}(t)}^{t} \xi_{\mu(s)}\left(-\lambda \sum_{j=1}^{m} \frac{q_{j}(t)}{e_{p}\left(\eta_{j}(t), t\right)}\right) \Delta s\right)}\right\}<1$,

where

$$
\begin{gathered}
E_{1}=\left\{\lambda>0: 1-\lambda \sum_{k=1}^{n+m} h_{k}(t) \mu(t)>0\right\}, E_{2}=\left\{\lambda>0: 1-\lambda \sum_{j=1}^{m} \frac{q_{j}(t)}{e_{p}\left(\eta_{j}(t), t\right)} \mu(t)>0\right\}, \\
h_{k}(t)=\left\{\begin{array}{cc}
p_{k}(t) & k=1,2, \ldots n \\
q_{k-n}(t) & k=1,2, \ldots m+n,
\end{array}\right.
\end{gathered}
$$




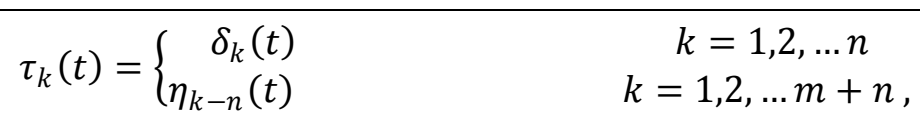

and $P=\sum_{i=1}^{n} p_{i}(t)$

then equation (26) is oscillatory.

\section{Proof:-}

Suppose that $x(\mathrm{t})$ is an eventually positive solution of equation (26) then there exist $T \epsilon\left[t_{0}, \infty\right)_{T}$ sufficiently large such that $x(t)>0, x\left(\delta_{i}(t)\right)>0$ for all $i=1,2, \ldots n, x\left(\eta_{j}(t)\right)>0$, for all $j=$ $1,2, \ldots m$, for all $\mathrm{t} \geq \mathrm{T}$. Then $x^{\Delta}(t)<0$ i.e, $x$ is non-increasing and consequently from (26) we have

$$
\mathrm{x}^{\Delta}(\mathrm{t})+\sum_{i=1}^{n} p_{i}(t) x\left(\delta_{i}(t)\right)+\sum_{j=1}^{m} q_{j}(t) x\left(\eta_{j}(t)\right)=0
$$

the above equation can be written in the form

$x^{\Delta}(t)+\sum_{k=1}^{n+m} h_{k}(t) x\left(\tau_{k}(t)\right)=0$,

where

and

$$
h_{k}(t)=\left\{\begin{array}{r}
p_{k}(t) \\
q_{k-n}(t)
\end{array} \quad \begin{array}{c}
k=1,2, \ldots n \\
k=1,2, \ldots m+n,
\end{array}\right.
$$

$$
\tau_{k}(t)=\left\{\begin{array}{r}
\delta_{k}(t) \\
\eta_{k-n}(t)
\end{array} \quad k=1,2, \ldots m+n .\right.
$$

But according to [1] all solutions of equation (27) are oscillatory if

$$
\lim _{t_{0 \rightarrow \infty}} \sup _{t>t_{0}} \sup _{\lambda \in E_{1}}\left\{\frac{\lambda \sum_{k=1}^{n+m} h_{k}(t)}{\sum_{k=1}^{n+m} h_{k}(t) \exp \left(-\int_{\tau_{k}(t)}^{t} \xi_{\mu(s)}\left(-\lambda \sum_{k=1}^{n+m} h_{k}(s)\right) \Delta s\right)}\right\}<1,
$$

which is a contradiction.

Suppose that $x(\mathrm{t})$ is an eventually negative solution of equation (26) then there exists $T \in\left[t_{0}, \infty\right)_{T}$ sufficiently large such that $x(t)<0, x\left(\delta_{i}(t)\right)<0$ for all $i=1,2, \ldots n, x\left(\eta_{j}(t)\right)<0$ for all $j=$ $1,2, \ldots m$. Take $y=-x$, then equation (26) takes the form

$\mathrm{y}^{\Delta}(\mathrm{t})+\sum_{i=1}^{n} p_{i}(t) y(\sigma(t))+\sum_{j=1}^{m} q_{j}(t) y\left(\eta_{j}(t)\right)=0$.

Let $P=\sum_{i=1}^{n} p_{i}(t)$. Multiplying equation (28) by $e_{p}\left(t, t_{0}\right)$ then, we have

i.e,

$$
\mathrm{y}^{\Delta}(\mathrm{t}) e_{P}\left(t, t_{0}\right)+P y(\sigma(t)) e_{P}\left(t, t_{0}\right)+\sum_{j=1}^{m} q_{j}(t) y\left(\eta_{j}(t)\right) e_{P}\left(t, t_{0}\right)=0,
$$

$\operatorname{let} z(t)=y(t) e_{P}\left(t, t_{0}\right)$

$$
\left(\mathrm{y}^{\Delta}(\mathrm{t}) e_{P}\left(t, t_{0}\right)\right)^{\Delta}++\left(\sum_{j=1}^{m} q_{j}(t) y\left(\eta_{j}(t)\right)\right) e_{p}\left(t, t_{0}\right)=0,
$$

i.e,

$$
z^{\Delta}(t)+e_{P}\left(t, t_{0}\right) \sum_{j=1}^{m} \frac{q_{j}(t) z\left(\eta_{j}(t)\right)}{e_{P}\left(\eta_{j}(t), t_{0}\right)}=0
$$

but all solutions are oscillatory if

$$
z^{\Delta}(t)+\sum_{j=1}^{m} \frac{q_{j}(t) z\left(\eta_{j}(t)\right)}{e_{P}\left(\eta_{j}(t), t\right)}=0
$$


$\lim _{t_{0 \rightarrow \infty}} \sup _{t>t_{0}} \sup _{\lambda \in E_{2}}\left\{\frac{\lambda \sum_{j=1}^{m} \frac{q_{j}(t)}{e_{p}\left(\eta_{j}(t), t\right)}}{\sum_{j=1}^{m} \frac{q_{j}(t)}{e_{p}\left(\eta_{j}(t), t\right)} \exp \left(\sum^{2}-\int_{\eta_{j}(t)}^{t} \xi_{\mu(s)}\left(-\lambda \sum_{j=1}^{m} \frac{q_{j}(t)}{e_{p}\left(\eta_{j}(t), t\right)}\right) \Delta s\right)}\right\}<1$,

which is a contradiction, the proof is complete.

\subsubsection{Remark}

we note that if $\mathrm{T}=\mathrm{R}$ equation (26) becomes

$\mathrm{x}^{\prime}(\mathrm{t})+\sum_{i=1}^{n} p_{i}(t) \max _{s \in\left[\delta_{i}(t), t\right]} x(s)+\sum_{j=1}^{m} q_{j}(t) x\left(\eta_{j}(t)\right)=0$.

Then, if

and,

$$
\liminf _{t \rightarrow \infty} \frac{\sum_{i=1}^{n} p_{i}(t) \int_{\delta_{i}(t)}^{t}\left(\sum_{i=1}^{n} p_{i}(s) d s\right)+\sum_{j=1}^{m} q_{j}(t) \int_{\eta_{j}(t)}^{t}\left(\sum_{j=1}^{m} q_{j}(s) d s\right)}{\sum_{i=1}^{n} p_{i}(t)+\sum_{j=1}^{m} q_{j}(t)}>\frac{1}{e},
$$

$$
\liminf _{t \rightarrow \infty} \frac{\sum_{j=1}^{m} q_{j}(t) e^{\int_{\eta_{j}(t)}^{t}\left(\sum_{i=1}^{n} p_{i}(s) d s\right)} \int_{\eta_{j}(t)}^{t}\left(\sum_{j=1}^{m} q_{j}(s) e^{\int_{\eta_{j}(s)}^{s}\left(\sum_{i=1}^{n} p_{i}(\xi) d \xi\right)} d s\right)}{\sum_{j=1}^{m} q_{j}(t) e^{\int_{\eta_{j}}^{t}(t)\left(\sum_{i=1}^{n} p_{i}(s) d s\right)}}>\frac{1}{e^{\prime}},
$$

then all solutions of equation (29) are oscillatory. Condition (30) is a new criteria for oscillation of equation (27).

\section{EXAMPLES}

In this section we give some examples to illustrate our main results.

\subsection{Example}

Take $\mathrm{T}=\mathrm{R}$, consider the equation

$x^{\prime}(t)-\frac{1}{t(t-1)} \max _{s \in[t-2, t]} x(s)=0$.

Here, $p(t)=\frac{1}{t(t-1)}, t>3$ is of one sign then by Theorem (2.1) equation (31) isnonoscillatory. In fact $x(t)=\frac{t-1}{t}$ is a nonoscillatory solution for this equation.

\subsection{Example}

Take $\mathrm{T}=\mathrm{R}$, consider the equation

$x^{\prime}(t)+\left(e^{-t}+1\right) e^{\frac{-3}{2}} \max _{s \in\left[t-\frac{3}{2}, t\right]} x(s)-e^{-\left(t+\frac{1}{2}\right)} \max _{s \in\left[t-\frac{1}{2}, t\right]} x(s)=0$.

Here, $p_{1}(t)=\left(e^{-t}+1\right) e^{\frac{-3}{2}}, p_{2}(t)=e^{-\left(t+\frac{1}{2}\right)}, \delta_{1}=\frac{3}{2}$ and $\delta_{2}=\frac{1}{2}$. It is clear that $\delta_{1}>\delta_{2}, p_{1}(t)>$ $p_{2}(t-1), t \geq 1$. Then according to the Remark (2.2.1) then every solution of this equation is oscillatory or tends to zero. In fact $x=e^{-t}$ is a solution which is tends to zero.

\subsection{Example}

Take $\mathrm{T}=\mathrm{R}$, consider the equation

$x^{\prime}(t)+\frac{1}{3} \max _{s \in[t-3, t]} x(s)+\frac{2}{3} \max _{s \in[t-2, t]} x(s)+\frac{2}{e} x(t-1)=0$.

Here, $p_{1}=\frac{1}{3}, p_{2}=\frac{2}{3}, \delta_{1}=3$ and $\delta_{2}=2$,

$$
\liminf _{t \rightarrow \infty} \frac{\frac{1}{3} \int_{t-3}^{t}\left(\frac{1}{3}+\frac{2}{3}\right) d s+\frac{2}{3} \int_{t-2}^{t}\left(\frac{1}{3}+\frac{2}{3}\right) d s+\frac{2}{e} \int_{t-1}^{t} \frac{2}{e} d s}{\frac{1}{3}+\frac{2}{3}+\frac{2}{e}}>\frac{1}{e},
$$

and 


$$
\lim _{t \rightarrow \infty} \frac{\frac{2}{e} e^{\int_{t-1}^{t}\left(\frac{1}{3}+\frac{2}{3}\right) d s} \int_{t-1}^{t} \frac{2}{e} e^{\int_{s-1}^{s}\left(\frac{1}{3}+\frac{2}{3}\right) d \xi} d s}{\frac{2}{e} e^{\int_{t-1}^{t}\left(\frac{1}{3}+\frac{2}{3}\right) d s}}>\frac{1}{e}
$$

then equation (33) is oscillatory.

\section{REFERENCES}

[1] HilgerS., Analysis on measure chains-A unified approach to continuous and discrete calculus, Results Math. 18 (1990), PP. 18-56,

[2] Kac V. andChueng P., Quantum Calculus, Universitext, Springer, New York, NY, USA, 2002.

[3] Bohner M., Peterson A., Dynamic Equations on Time Scales: An Introduction with Applications, Birkhäuseruser, Boston, 2001.

[4] Bohner M. and Peterson A., Advances in Dynamic Equations on Time Scales, Birkhäuseruser, Boston 2003.

[5] Bainov D. D. andZaharievA. I., Oscillating and asymptotic properties of a class of functional differential equations with maxima, Czechoslovak Math. J.. 34, pp.247-251, (1984).

[6] Bainov D. D. and G. HristovaSnezhana, Differential Equations with Maxima,Taylor and Francis Group, 2011.

[7] Zhang B. G. and ZhangGuang, Qualitative properties of functional differential equations with "maxima", Rocky Mountain J. Math. 29, pp. 357-367, (1999).

[8] Agwo H. A., On the oscillation of first order delay dynamic equations with variable coefficients, Rocky Mountain J. Math. 38, pp.1-18, (2008).

\section{AUTHORS' BIOGRAPHY}

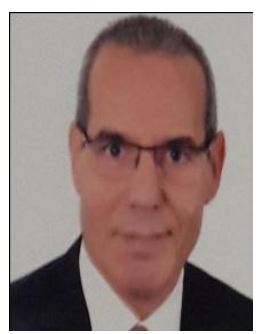

H. A. Agwa is professor of pure mathematics and vice dean for Education and Student Affairs at faculty of education Ain shams university. He has published several papers in many international journals. His main research interests are: functional differential equations, dynamic equations, time scales, and inequalities.

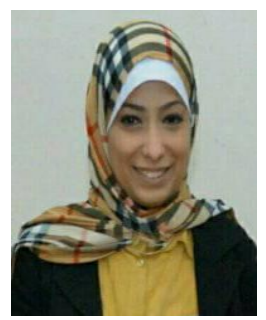

Esraa Magdy is demonstrator in mathematics department, Faculty of Education, Ain shams university.

Citation: H. A. Agwa, E. Magdy, "Oscillation Criteria for First Order Dynamic Equations with Maxima on Time Scales ", International Journal of Scientific and Innovative Mathematical Research, vol. 5, no. 10, p. 1 8, 2017., http://dx.doi.org/10.20431/2347-3142.0510001

Copyright: () 2017 Authors. This is an open-access article distributed under the terms of the Creative Commons Attribution License, which permits unrestricted use, distribution, and reproduction in any medium, provided the original author and source are credited. 